\title{
Amyotrophe Lateralsklerose - Herausforderungen von pflegenden Angehörigen
}

\section{Amyotrophic Lateral Sclerosis - Challenges of Family Caregivers}

Autoren

Lisa Baumann', Michael Klösch', Markus Greger², Anna Dieplinger', Stefan Lorenzl'

Institute

1 Paracelsus Medizinische Privatuniversität Salzburg

2 Vinzentinum Linz, Langgasse 19, 4020 Linz

Schlüsselwörter

Amyotrophe Lateralsklerose, Motoneuronerkrankung, Verhalten, Kognition, Belastung pflegender Angehöriger

Key words

amyotrophic lateral sclerosis, motor neuron disease, behaviour, cognition, caregiver burden

eingereicht 30.04 .2019

akzeptiert $\quad 17.05 .2019$

Bibliografie

DOI https://doi.org/10.1055/a-0934-6163

Fortschr Neurol Psychiatr 2019; 87: 476-482

(c) Georg Thieme Verlag KG Stuttgart · New York

ISSN 0720-4299

Korrespondenzadresse

Lisa Baumann

Paracelsus Medizinische Privatuniversität

Strubergasse 21

5020 Salzburg

Tel.: 06803286563

Fax: 074848221

E-Mail: lisa.baumann@stud.pmu.ac.at

\section{ZUSAMMENFASSUNG}

Hintergrund Bei Patientinnen und Patienten mit amyotropher Lateralsklerose (ALS) kann es im Krankheitsverlauf zum Auftreten von Veränderungen im Verhalten und in der Kognition kommen. Ein Teil der Betroffenen entwickelt eine Frontotemporale Demenz. Für die pflegenden Angehörigen stellen diese Veränderungen eine zusätzliche Herausforderung dar.

Ziel der Arbeit Beschreibung der Auswirkungen von krankheitsbedingten Veränderungen in Kognition und Verhalten auf das emotionale Befinden der pflegenden
Angehörigen und die genaue Erörterung der resultierenden Belastungen.

Methodik In der vorliegenden Arbeit wurde mittels verschiedener Suchbegriffe in diversen Datenbanken recherchiert. Im Ergebnisteil erfolgt die Synthese der eingeschlossenen zehn Studien zur Beantwortung des Forschungsinteresses. Die Diskussion umfasst die weitere Auseinandersetzung mit der Thematik unter Einbeziehung ergänzender Literatur.

Ergebnisse Veränderungen im Verhalten und in der Kognition können die Entstehung einer Angst- und Depressionssymptomatik bei pflegenden Angehörigen begünstigen. Des Weiteren verweisen die Ergebnisse der Studien auf einen Zusammenhang von Apathie, Enthemmung und exekutiven Dysfunktionen mit einem gesteigerten Belastungsempfinden bei pflegenden Angehörigen.

Schlussfolgerung Veränderungen im Verhalten und in der Kognition bei Patienten mit amyotropher Lateralsklerose wirken sich negativ auf das emotionale Befinden der pflegenden Angehörigen aus und tragen zu einem erhöhten Belastungsempfinden bei. Hinsichtlich dieser krankheitsbedingten Veränderungen bedarf es einer frühzeitigen und ausführlichen Aufklärung der Betroffenen.

\section{ABSTRACT}

Background Patients who suffer from amyotrophic lateral sclerosis sometimes show pathological changes in behaviour and cognition. Some even develop frontotemporal dementia. The family caregivers of the patients are faced with additional challenges.

Aim To describe the effect of changes in behaviour and cognition on the emotional state and the perceived stress and burdens of family caregivers.

Method Different search terms were used for searching in several databases. Ten trials were included in the study. Further examinations of this topic on the basis of additional literature are described in the discussion section.

Results Changes in behaviour and cognition can lead to symptoms of anxiety and depression in family caregivers. The findings also show a correlation between apathy, disinhibition and executive dysfunction combined with an increase in caregiver burden. 
Conclusion Changes in behaviour and cognition in patients with ALS have a negative impact on the emotional state of family caregivers and contribute to increased feelings of being burdened. Early and detailed information concerning possible disease-induced changes is necessary.

\section{Einleitung}

Die amyotrophe Lateralsklerose (ALS) ist eine neurodegenerative Erkrankung, an der jährlich etwa 1-2 Personen auf 100.000 Menschen neu erkranken [1]. ALS wurde erstmals vom französischen Neurologen Jean-Martin Charcot im Jahre 1874 beschrieben [2] und erlangte Aufmerksamkeit in der breiten Öffentlichkeit vor allem durch betroffene Persönlichkeiten wie den amerikanischen Baseballspieler Lou Gehrig oder den britischen Astrophysiker Stephen Hawking [3] und zuletzt durch Aktionen wie die „Ice bucket challenge“. Ab dem Auftreten der ersten Symptome beträgt die durchschnittliche Überlebensrate 3,5 Jahre. Durch den raschen Krankheitsverlauf und die damit einhergehende kurze Lebenserwartung ergibt sich eine niedrige Prävalenz von 3-8 Betroffenen auf 100000 [4]. Die Krankheit nimmt nicht nur auf die Betroffenen, sondern auch auf die gesamte Familienstruktur großen Einfluss. Allgemein werden an ALS erkrankte Personen hauptsächlich von ihren Ehe- bzw. Lebenspartnern oder Kindern zu Hause gepflegt [5]. Nicht nur die Betroffenen, sondern auch deren pflegende Angehörige werden vor große physische und psychische Herausforderungen gestellt [6]. Neben den bekannten körperlichen Veränderungen treten bei einem Teil der Betroffenen Verhaltensauffälligkeiten bzw. Wesensveränderungen auf. Dabei können geringfügige Abweichungen vom Normalverhalten bis hin zu Symptomen einer Frontotemporalen Demenz (FTD) bemerkt werden. Die damit einhergehenden Veränderungen wie Apathie, Verlust von Interesse, Empathie und sozialen sowie emotionalen Beziehungen stellen vor allem für die pflegenden Angehörigen eine psychische Herausforderung dar [7]. Darüber hinaus werden Veränderungen in Kognition, Verhalten und Emotionalität, die durch die frontotemporale Degeneration bei ALS entstehen können, beschrieben [8]. Zusätzlich zeigt sich eine verminderte Wahrnehmung der eigenen Verhaltensveränderungen bei ALS-Patientinnen und -Patienten mit frontotemporalen Symptomen. Gerade dieses fehlende Einsichts- und Urteilsvermögen ist ein weiterer Belastungsfaktor für die pflegenden Angehörigen [9]. Zudem gelingt es vielen ALS-Patientinnen und -Patienten nicht, mit ihrer Launenhaftigkeit, Frustration und Ärger adäquat umzugehen. In der Folge kommt es zu aggressiven Tendenzen gegenüber pflegenden Angehörigen. Weitere mögliche Veränderungen im Denken und Handeln sind soziale Enthemmung, abnormales Essverhalten, Reizbarkeit, vermindertes Urteilsvermögen und Impulsivität [8]. Zusätzlich zu den oben angeführten emotionalen Belastungen stellt die Pflege von an ALS erkrankten Personen einen hohen physischen Anspruch an deren pflegende Angehörige. Häufig müssen bereits vor Eintritt in die letzte Phase der Erkrankung entscheidende Aktivitäten des täglichen Lebens wie Körperpflege, Mobilisation und Ernährung vollständig übernommen werden. Das stellt insbesondere für ältere und/oder körperlich eingeschränkte Angehörige ein zusätzliches Problem dar [10].
Das Ziel der vorliegenden Literaturarbeit ist es, anhand einer systematischen Literaturrecherche die Auswirkungen von krankheitsbedingten Veränderungen in Kognition und Verhalten bei Patientinnen und Patienten mit amyotropher Lateralsklerose auf das emotionale Befinden der pflegenden Angehörigen zu beschreiben und resultierende Belastungen zu erörtern.

\section{Material und Methoden}

Es wurde eine systematische Literaturrecherche mit dem Publikationszeitraum von 2006 bis 2018 durchgeführt. Die systematische Online-Recherche erfolgte in den Datenbanken CINAHL (Cumulative Index to Nursing and Allied Health Literature), PubMed (U.S. National Library of Medicine) und SpringerLink mit folgenden Suchbegriffen: „amyotrophic lateral sclerosis“, „motor neuron disease“, „caregiver burden“, „behaviour“ und „cognition“. „AND“ und „OR“ dienten als Operatoren zur exakten Eingrenzung. Es wurden 191 Treffer erzielt. Die dabei erhaltenen Arbeiten wurden nach dem EMED-Format analysiert. Daraus ergaben sich 29 Studien. Mittels definierter Ein- und Ausschlusskriterien ( $\triangleright$ Tab. 1) konnten zwölf Publikationen als relevant beurteilt werden, davon waren zwei Doppeltreffer; somit konnten zehn Studien eingeschlossen werden ( $\triangleright$ Abb. 1 ).

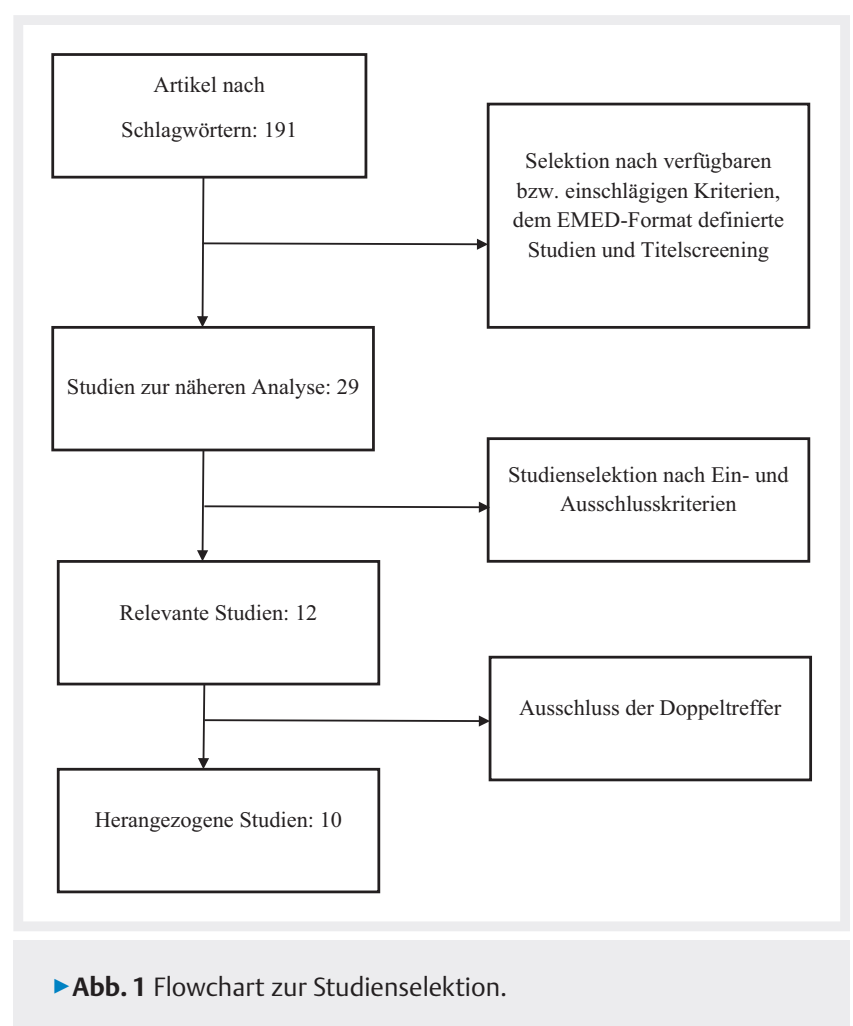


-Tab. 1 Ein- und Ausschlusskriterien.

\begin{tabular}{|c|c|}
\hline Einschlusskriterien & Ausschlusskriterien \\
\hline $\begin{array}{l}\text { Studien, welche zwischen } 2006 \\
\text { und } 2018 \text { publiziert wurden }\end{array}$ & $\begin{array}{l}\text { Studien, welche vor dem Jahr } \\
2006 \text { publiziert wurden }\end{array}$ \\
\hline $\begin{array}{l}\text { Veränderung in Kognition } \\
\text { und / oder Verhalten }\end{array}$ & $\begin{array}{l}\text { Keine Veränderung in Kognition } \\
\text { und / oder Verhalten }\end{array}$ \\
\hline $\begin{array}{l}\text { Betroffene mit } \\
\text { amyotropher Lateralsklerose }\end{array}$ & $\begin{array}{l}\text { Betroffene mit anderen neurolo- } \\
\text { gischen oder demenziellen } \\
\text { Erkrankungen / } \\
\text { Begleiterkrankungen }\end{array}$ \\
\hline $\begin{array}{l}\text { Angehörige als Hauptpflegeper- } \\
\text { son (Lebenspartner, Kinder, } \\
\text { Eltern) }\end{array}$ & $\begin{array}{l}\text { Hauptpflege durch andere Per- } \\
\text { sonen bzw. Institutionen }\end{array}$ \\
\hline Pflegende Angehörige > 18 Jahre & Pflegende Angehörige $<18$ Jahre \\
\hline Gliederung im EMED-Format & Kein bestehendes EMED-Format \\
\hline $\begin{array}{l}\text { Quantitative und qualitative Stu- } \\
\text { dien, Literaturstudien, Meta- } \\
\text { Analysen und -Synthesen, } \\
\text { Fallstudien }\end{array}$ & $\begin{array}{l}\text { Wissenschaftliche Artikel, Bache- } \\
\text { lor- und Masterarbeiten, } \\
\text { Dissertationen }\end{array}$ \\
\hline
\end{tabular}

\section{Ergebnisse}

Die mithilfe der systematischen Literaturrecherche ausgewählten Studien für den Ergebnisteil werden in der $>$ Tab. 2 mit einer Kurzbeschreibung angeführt. Im Speziellen werden Autor, Studiendesign, Methodik und die Ergebnisse kurz zusammengefasst.

\section{Veränderungen in Kognition und Verhalten}

Caga et al. beschreiben bei 33\% der Erkrankten Veränderungen in der Kognition und bei $53 \%$ Verhaltensveränderungen [14]. Bei ALS-Patientinnen und -Patienten kann es zu einer unterschiedlich stark ausgeprägten Form der Frontotemporalen Demenz kommen. Etwa $5 \%$ der betroffenen Personen weisen in den Bereichen Kognition und Verhalten eindeutige Symptome der Demenz auf [12]. Es zeigt sich eine unterschiedliche Wahrnehmung der Veränderungen in Kognition und Verhalten zwischen erkrankten Personen und ihren pflegenden Angehörigen. Während laut Angaben der Angehörigen 48,6\% der Erkrankten pathologische Verhaltensweisen aufweisen, zeigte die Selbsteinschätzung der ALS-Patientinnen und -Patienten nur 12,9\% [15]. Cui et al. erwähnen bei $24,6 \%$ der ALS-Patientinnen und -Patienten eine abnormal gesteigerte Erregbarkeit im Verhalten, bei 20,0\% der Betroffenen Wortkargheit und bei 15,4\% der Erkrankten übermäBige Einsichtslosigkeit [16]. Darüber hinaus treten Veränderungen wie Apathie, Enthemmung und exekutive Dysfunktionen bei den Betroffenen auf. Laut Angaben der pflegenden Angehörigen zeigen $55,7 \%$ der Patientinnen und Patienten Symptome der Apathie. Exekutive Dysfunktionen wurden bei $45,7 \%$ und Symptome der Enthemmung bei $25,7 \%$ der Erkrankten beschrieben [15]. Abnormale Verhaltensweisen, Essensgewohnheiten und stereotype oder motorische Bewegungen wurden von pflegenden Angehörigen bei mehr als $50 \%$ der untersuchten ALS-Patientinnen und -Patienten festgestellt. $10-30 \%$ davon sind den
Ausprägungsgraden moderat bis schwer zugeordnet. Mit einem Auftreten bei ca. 80 \% der ALS-Erkrankten stellt Apathie die häufigste Verhaltensveränderung dar. $40 \%$ davon werden von den jeweiligen pflegenden Angehörigen als moderate bis schwere Ausprägung beschrieben [17].

\section{Belastungen durch Verhaltensveränderung}

Das krankheitsbedingte Auftreten von Verhaltensveränderungen steht in engem Zusammenhang mit einem erhöhten Belastungsempfinden bei pflegenden Angehörigen. Ein Drittel der von pflegenden Angehörigen wahrgenommenen Belastung entsteht durch diese Verhaltensveränderungen [13]. Bezüglich Abweichungen in Erinnerung und Orientierung, Alltagsfertigkeiten und Motivation konnte ein Zusammenhang zwischen diesen Veränderungen und einem erhöhten Belastungsempfinden hergestellt werden [11]. Insbesondere die Apathie als Symptom der ALS trägt wesentlich dazu bei [18]. Je stärker die Ausprägung der Apathie war, desto belastender empfanden die Angehörigen die Versorgung [14]. Ebenso spielt eine gesteigerte Enthemmung bei Patientinnen und Patienten eine Rolle. Auch exekutive Fehlfunktionen konnten als Auslöser für ein erhöhtes Belastungsempfinden bei pflegenden Angehörigen identifiziert werden [13]. Es zeigte sich ein signifikanter Zusammenhang zwischen exekutiven Dysfunktionen und dem Gesamtbelastungsempfinden [5, 18]. Pflegende Angehörige von ALS-Patientinnen und -Patienten mit abnormalen Verhaltensweisen haben laut Lillo et al. ein 1,4-mal höheres Risiko für ein gesteigertes Belastungsempfinden als Betreuer von Betroffenen ohne verändertes Verhalten [17]. Durch das Auftreten von Verhaltensveränderungen kommt es zu einer gesteigerten sozialen und emotionalen Belastung der pflegenden Partner und Kinder [19]. Auch das pathologische Lachen oder Weinen stellt häufig eine Belastung für die Angehörigen dar und wird als psychosozialer Stressor empfunden [20].

\section{Belastungen durch Kognitionsveränderung}

Eine Veränderung der kognitiven Funktion wird ebenfalls als Belastungsfaktor empfunden [12]. Kognitive Symptome bei Patientinnen und Patienten wirken sich negativ auf die entwicklungsbedingte, die physische, die soziale und die emotionale Belastung aus [16]. Bock et al. verweisen auf einen signifikanten Zusammenhang zwischen veränderter Aufmerksamkeit sowie Konzentration und einem erhöhten Gefühl der Belastung bei pflegenden Angehörigen [12].

\section{Belastungen durch Angst bei pflegenden Angehörigen}

$16 \%$ der befragten pflegenden Angehörigen leiden an moderater bis schwerer Angstsymptomatik [17]. Watermeyer et al. beschreiben einen signifikanten Zusammenhang zwischen dem Auftreten von anormalen Verhaltensweisen wie Apathie, Enthemmung und exekutiven Dysfunktionen bei ALS-Patientinnen und -Patienten und einem gesteigerten Angstgefühl bei pflegenden Angehörigen. Eine höhere emotionale Labilität bei Erkrankten führt zu einem 
> Tab. 2. Studienbeschreibung.

\begin{tabular}{|c|c|c|}
\hline Autorinnen / Jahr & Design und Assessmentinstrumente & Ergebnisse \\
\hline Andrews et al. (2016) & $\begin{array}{l}\text { Fall-Kontroll Studie } \\
\text { - Cambridge Behavioural Inventory (CBI-R) } \\
\text { - ALS Functional Rating Scale (ALSFRS-R) } \\
\text { - Zarit Burden Inventory (ZBI) }\end{array}$ & $\begin{array}{l}\text { Korrelation zW. CBI-R und ZBI } \\
\text { allg. Korrelation zwischen Verhaltensveränderung und } \\
\text { Belastung }(r=0.657, p<0.001) \\
\text { Abweichung in Erinnerung und Orientierung }(r=0.625 \text {, } \\
p<0.001) \text {, Alltagsfertigkeiten }(r=0.407, p<0.009) \text { und } \\
\text { Motivation ( } r=0.426, p<0.006) \text { wirkt negativ auf Belas- } \\
\text { tung pflegender Angehöriger }\end{array}$ \\
\hline Bock et al. (2016) & $\begin{array}{l}\text { Querschnittstudie } \\
\text { - ALS Cognitive-Behavioural Screen (ALS CBS) } \\
\text { - ALS Functioning Rating Scale-Revised (ALSFRS-R) } \\
\text { - Caregiver Burden Scale (CGBS) }\end{array}$ & $\begin{array}{l}\text { CBS Cognitive Score }(p<0.05) \text { und CBS Behavioral Score } \\
(p<0.001) \text { werden mit erhöhtem Belastungsempfinden } \\
\text { der Angehörigen }(C G B S) \text { assoziiert. } \\
\text { - verminderte Aufmerksamkeit }(r=-0.25, p=0.03) \\
\text { - verminderte Konzentration }(r=-0.25, p=0.03)\end{array}$ \\
\hline Burke et al. (2015) & $\begin{array}{l}\text { Querschnittstudie } \\
\text { - Zarit Burden Interview (ZBI) } \\
\text { - Frontal Systems Behavioral Scale (FrSBe) } \\
\text { - Hospital Anxiety and Depression Scale (HADS-T) } \\
\quad \text { Depression (HADS-D), Anxiety (HADS-A) }\end{array}$ & $\begin{array}{l}\text { Ursachen für Belastung und allg. Leidensgefühl } \\
\text { - Apathie }(r=0.390, p=0.03) \\
\text { - Enthemmung }(r=0.530, p=0.002) \\
\text { - Exekutive Funktionen }(r=0.372, p=0.039) \\
\text { - Verhalten trägt zu } 31 \% \text { zu Belastungsempfinden bei } \\
\quad \text { pfl. Angehörigen bei }(r=0.563, p=0.015) \\
\text { - HADS-T trägt zu } 38.5 \% \text { zu Belastung bei }(r=0.621 \text {, } \\
\quad p<0.000)\end{array}$ \\
\hline Caga et al. (2018) & $\begin{array}{l}\text { Querschnittstudie } \\
\text { - ALS Functional Rating Scale (ALSFRS-R) } \\
\text { - Hospital Anxiety and Depression Scale (HADS) } \\
\text { - Apathy Evaluation Scale (AES) } \\
\text { - Zarit Burden Inventory (ZBI) }\end{array}$ & $\begin{array}{l}\text { Unterschied zwischen mehr und weniger belasteten Pfle- } \\
\text { genden in Bezug auf Symptome der Apathie bei den } \\
\text { Erkrankten (AES) } \\
\text { - Apathie im Verhalten }(p=0.011) \\
\text { - unspezifische Apathie }(p=0.010)\end{array}$ \\
\hline Chio et al. (2010) & 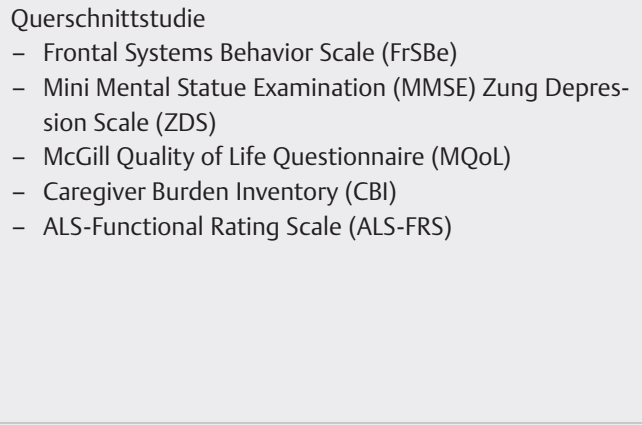 & $\begin{array}{l}\text { signifikante Korrelation zwischen Depression der pflegen- } \\
\text { den Angehörigen und } \\
\text { - FrSBe Skala der Patienten }(r=0.386, p=0.001) \\
\text { - Apathie }(r=0.353, p=0.003) \\
\text { - Exekutive Fehlfunktionen }(r=0.387, p=0.001) \\
\text { sign. Korrelation zW. CBI und } \\
\text { - FrSBe Skala der Patienten (Fremdeinschätzung) } \\
\quad(r=0.384, p=0.001) \\
\text { - Apathie }(r=0.313, p=0.008) \\
\text { - Exekutive Fehlfunktionen }(r=0.435, p=0.0002) \\
\text { Korrelation zw. Lebensqualität von pflegenden Angehöri- } \\
\text { gen und FrSBe }(r=-0.387, p<0.001)\end{array}$ \\
\hline Cui et al. (2015) & $\begin{array}{l}\text { Querschnittstudie } \\
\text { - ALS Functioning Rating Scale Revised (ALSFRS-R) } \\
\text { - Frontal Behavioral Inventory-ALS (FBI-ALS) } \\
\text { - Frontal Assessment Battery (FAB) } \\
\text { - Caregiver Burden Inventory (CBI) }\end{array}$ & $\begin{array}{l}\text { sign. Korrelation zw. FAB Score (Kognition) und total CBI } \\
(r=-0.411, p<0.01) \\
\text { - FAB Score / entwicklungsbedingte Belastung }(r=-0.366 \text {, } \\
\quad p<0.01) \\
\text { - FAB Score / physische Belastung }(r=-0.358, p<0.01) \\
\text { - FAB Score / soziale Belastung }(r=-0.295, p<0.05) \\
\text { - FAB Score / emotionale Belastung }(r=-0.278, p<0.05)\end{array}$ \\
\hline Goldstein et al. (2006) & $\begin{array}{l}\text { Kohortenstudie } \\
\text { - Emotional Lability Questionnaire (ELQ) } \\
\text { - Anxiety And Depression Scale (HADS) } \\
\text { - Strain Scale of Morris } \\
\text { - Caregiver Burden Inventory (CBI) } \\
\text { - Carer Outcome = (HADS Score + strain + burden score) }\end{array}$ & $\begin{array}{l}\text { sign. Korrelation zw. ELQ und Carer Outcome } \\
\text { - 1. Zeitpunkt.: } r=0.464, p=0.003 \\
-\quad \text { 2. Zeitpunkt: } r=0.465, p=0.019 \\
-\quad \text { 3. Zeitpunkt: } r=0.535, p=0.012\end{array}$ \\
\hline Lillo et al. (2012) & $\begin{array}{l}\text { Querschnittstudie } \\
\text { - ALS Functioning Rating Scale - Revised (ALSFRS-R) } \\
\text { - Cambridge Behavioral Inventory Revised (CBI-R) } \\
\text { - Depression Anxiety Stress scale (DASS 21) } \\
\text { - Zarit Burden Interview (ZBI) }\end{array}$ & 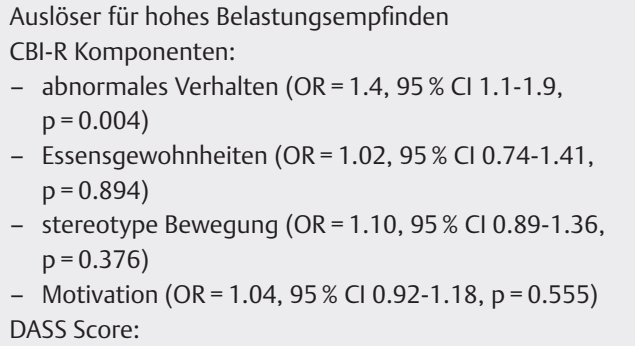 \\
\hline
\end{tabular}


-Tab. 2. Fortsetzung ...

\begin{tabular}{|c|c|c|}
\hline Autorlnnen / Jahr & Design und Assessmentinstrumente & Ergebnisse \\
\hline & & $\begin{array}{l}\text { - Depression }(O R=1.07,95 \% \mathrm{Cl} 0.96-1.19, p=0.243) \\
\text { - Angst }(O R=0.93,95 \% \mathrm{Cl} 0.86-1.00, p=0.051) \\
\text { - Stress }(O R=1.1,95 \% \mathrm{Cl} 1.0-1.3, p=0.024)\end{array}$ \\
\hline Tremolizzo et al. (2016) & $\begin{array}{l}\text { Querschnittstudie } \\
\text { - } \text { ALS-Cognitive Behavioural Screen } \\
\text { (ALSCBS-ci) -Kognition } \\
\text { (ALSCBS-bi) -Verhalten. } \\
\text { - Frontal Assessment Battery } \\
\text { - Beck's Depression Inventory } \\
\text { (BDI-p) Patienten, (BDI-c) Pflegende } \\
\text { - Caregiver Burden Inventory (CBI) }\end{array}$ & $\begin{array}{l}\text { ALSCBS-bi korreliert mit CBI Unterkategorie } \\
\text { - soziale Belastung }(r=-0.253, p<0.03) \\
\text { - emotionale Belastung }(p=0.069) \\
\text { BDI-c score korreliert mit } \\
\text { - ALSCBS-bi }(r=-0.269, p<0.02)\end{array}$ \\
\hline $\begin{array}{l}\text { Watermeyer et al. } \\
\text { (2015) }\end{array}$ & $\begin{array}{l}\text { Querschnittstudie } \\
\text { - Hospital Anxiety and Depression Scale (HADS) } \\
\text { - Zarit Burden Interview (ZBI) } \\
\text { - ALS Functioning Rating Scale - Revised (ALSFRS-R) } \\
\text { - Frontal Systems Behavioural Scale (FrSBe) } \\
\text { - Emotional Lability Questionnaire (ELQ) }\end{array}$ & $\begin{array}{l}\text { sign. Korrelation von ZBI score } \\
\text { - FrSBe Apathie }(r=0.63, p<0.001) \\
\text { - FrSBe Enthemmung }(r=0.51, p=0.003) \\
\text { - FrSBe exekutive Fehlfunktionen }(r=0.51, p=0.003) \\
\text { - FrSBe Total }(r=0.69, p<0.001) \\
\text { sign. Korrelation von HADS-R D } \\
\text { - mit FrSBe Apathie }(r=0.43, p=0.01) \\
\text { - mit FrSBe Total }(r=0.37, p=0.04) \\
\text { sign. Korrelation von HADS-R A } \\
\text { - mit FrSBe Total }(r=0.40, p=0.02) \\
\text { - ELQ Total }(r=0.36, p=0.03)\end{array}$ \\
\hline
\end{tabular}

vermehrten Gefühl der Angst bei pflegenden Angehörigen [18]. Das gesteigerte Angstempfinden der Pflegenden erhöht wiederum das Risiko für das Entstehen weiterer Belastungen, insbesondere Depressionen [17].

\section{Belastungen durch Depression bei pflegenden Angehörigen}

$31 \%$ der befragten pflegenden Angehörigen in der Studie von Lillo et al. hatten eine moderate bis sehr schwere Depression, während $9 \%$ eine leichtere Form der Depression beschrieben [17]. Das Auftreten von Angst und Depression bei pflegenden Angehörigen führt in einem Teufelskreis zu einem gesteigerten Belastungsempfinden [13]. Bezüglich des Auftretens einer Depression bei den Pflegenden wurde ein signifikanter Zusammenhang mit den Symptomen Apathie, Enthemmung und exekutiven Dysfunktionen bei den Betroffenen beschrieben [18]. Auch exekutive Dysfunktionen und die damit verbundenen Verhaltensänderungen werden im Zusammenhang mit depressiven Zustandsbildern bei pflegenden Angehörigen genannt [15, 19].

\section{Diskussion}

Die Literaturrecherche hat gezeigt, dass die Belastungen von Angehörigen von ALS-Patientinnen und -Patienten sehr hoch sein können. Die Gründe dafür sind vielfältig und liegen nicht selten in Veränderungen des Verhaltens der Erkrankten. Aber auch Angst und Depression bei den pflegenden Angehörigen sind häufig auftretende und belastende Symptome. Während nach der aktuellen
Studienlage nur eine kleine Gruppe der ALS-Patientinnen und -Patienten eine klinisch definierte Frontotemporal-Demenz entwickelt, können bei einer weitaus größeren Anzahl leichte bis mittelschwere Veränderungen in den Bereichen der Kognition und des Verhaltens festgestellt werden [9]. Dies sind insbesondere Apathie, Enthemmung und exekutive Dysfunktionen [15]. Es konnte gezeigt werden, dass diese Symptome von den Angehörigen und ALS-Erkrankten unterschiedlich wahrgenommen werden [5, 9]. Die mangelnde Einsicht der Patientinnen und Patienten bezüglich der Veränderungen im Verhalten und Denken stellt für die pflegenden Angehörigen nicht selten eine große Herausforderung dar [9]. In diesem Zusammenhang ist es allerdings interessant, dass lediglich 37,8\% der Pflegenden eine zusätzliche Unterstützung durch externe Personen beanspruchen, beispielsweise durch mobile Hilfsdienste. Die physische und emotionale Belastung bei pflegenden Angehörigen war signifikant höher als bei Pflegenden, die keine zusätzliche Unterstützung in Anspruch nahmen. Etwa ein Drittel der pflegenden Angehörigen nahm an Selbsthilfegruppen teil. In dieser Gruppe fanden sich insbesonders diejenigen Pflegenden, die erkrankte Angehörige mit ausgeprägten Symptomen und einem signifikant höheren Auftreten von auffälligem Verhalten betreuten [21]. Die progredient fortschreitende Krankheit mit fehlenden erfolgsversprechenden Therapiemaßnahmen führt zu Stressbelastung und Frustration bei pflegenden Angehörigen [22].

Da Angehörige in vielen Fällen unzureichend informiert und unerwartet von den Symptomen der ALS getroffen werden, kann der Bedarf einer ausführlichen Aufklärung bezüglich des Auftretens von Veränderungen in Verhalten und Kognition als wichtiges Ergebnis dieser Literaturrecherche beschrieben 
werden [23]. Speziell am Beginn der Erkrankung sind für die Pflegenden psychologische Bedürfnisse, Kommunikation, Information und die Teilnahme am sozialen Leben von großer Bedeutung [24]. Watermeyer et al. beschreiben einen ausgeprägten Zusammenhang zwischen dem Auftreten von Apathie, Enthemmung und exekutiven Dysfunktionen bei ALS-Patientinnen und -Patienten und Symptomen der Angst bei pflegenden Angehörigen [18]. Häufig vermindern die Verhaltensveränderungen der Erkrankten die Lebensqualität der pflegenden Angehörigen [15]. Als belastend empfinden Angehörige vor allem die Unsicherheit bezüglich des weiteren Krankheitsverlaufs und -zustands der/des Betroffenen sowie der ungewissen emotionalen und finanziellen Zukunft [25]. Symptome der Frontotemporalen Demenz mit Veränderungen in Verhalten, Denken, Empathie und Emotion stellen nicht nur physische, sondern auch psychische Anforderungen an die pflegenden Angehörigen [22]. Bedingt durch die Verhaltensveränderungen im weiteren Krankheitsverlauf, haben die Pflegenden ein erhöhtes Risiko, an einer Depression zu erkranken [15, 18]. Rabkin et al. beschreiben bei bereits unter einer Depression leidenden pflegenden Angehörigen im Vergleich zu nicht depressiven Personen ein signifikant höheres Belastungsempfinden [26]. Dazu wurde in der Studie von Pagnini et al. Aufmerksamkeit („mindfulness“) in Bezug auf die Entwicklung neuer Strategien zur Problemlösung bei pflegenden Angehörigen als protektiver Faktor für das Entstehen von Belastungen beschrieben. Eine gesteigerte Aufmerksamkeit hilft den Pflegenden, negative Emotionen, Belastungen und Symptome der Angst und Depression besser zu bewältigen [27]. Allgemein wird der psychische und physische Gesundheitszustand der Pflegenden im Vergleich zur Gesamtbevölkerung als deutlich schlechter beschrieben [28]. Oft werden die eigenen Bedürfnisse der Pflegenden zugunsten der Versorgung der Betroffenen in den Hintergrund gestellt [29]. Hinzu kommt die fehlende Unterstützung [30]. Es fällt den Pflegenden oftmals schwer, Hilfeleistungen durch Familie und Freunde zu erbitten oder anzunehmen. Im Gespräch mit den Angehörigen sollten daher frühzeitig Informationen bezüglich Unterstützungsmöglichkeiten durch mobile Hauskrankenpflege, spezielle Langzeitpflege-Einrichtungen oder mobile Hospiz- und Palliativteams gegeben werden [22].

Es ist allerdings wichtig, an dieser Stelle nochmals festzustellen, dass die Evaluierung der Verhaltensveränderungen in den meisten Studien durch pflegende Angehörige erfolgte. Möglicherweise nehmen die Angehörigen, bedingt durch die Belastung der Pflege, die Veränderungen in Kognition und Verhalten stärker wahr, als sie eigentlich sind. Umgekehrt können bereits gewohnte Verhaltensweisen übersehen werden und weniger Beachtung finden. Auch durch die verschiedenen Assessmentinstrumente zur Erhebung von Verhaltens- und Kognitionsveränderungen sowie die Belastungswahrnehmung ist die Vergleichbarkeit der Ergebnisse eingeschränkt. Die einbezogenen Studien wurden in verschiedenen Ländern durchgeführt. Die Wahrnehmung einer Belastung durch die Pflege eines Angehörigen ist u.a. abhängig vom jeweiligen individuellen kulturellen Hintergrund. Daher muss die Auslegung der Ergebnisse der vorliegenden Literaturstudie auf Patientinnen und Patienten sowie pflegende Angehörige in Deutschland und Österreich unter diesem Aspekt als limitiert betrachtet werden.

\section{Schlussfolgerung}

Aus den Ergebnissen der evaluierten Studien ist der Zusammenhang zwischen dem Auftreten von krankheitsbedingten Veränderungen in Verhalten und Kognition bei Patientinnen und Patienten mit ALS und einem gesteigerten Belastungsempfinden bei deren pflegenden Angehörigen ersichtlich. In weiterer Folge führt dies zu den Symptomen Angst und Depression sowie zu einer insgesamt verminderten Lebensqualität bei Pflegenden. In der klinischen Kommunikation mit pflegenden Angehörigen müssen die physischen und psychischen Herausforderungen bedingt durch Veränderungen in Kognition und Verhalten - eine intensivere Beachtung finden. Eine frühzeitige Aufklärung bereits zu Beginn der Erkrankung bildet die Grundvoraussetzung für eine adäquate Unterstützung der Erkrankten und pflegenden Angehörigen im fortschreitenden Krankheitsverlauf. Weitere Forschungsarbeiten zu den Themen Angehörigenedukation und bestmögliche emotionale und pflegerische Unterstützung sind nach eingehender Auseinandersetzung mit der Thematik erforderlich.

Interessenkonflikt

Die AutorInnen erklären, dass keine Interessenkonflikte bestehen.

Literatur

[1] Deutsche Gesellschaft für Muskelkranke. Amyotrophe Lateralsklerose (ALS). Im Internet: https://www.dgm.org/muskelerkrankungen/amyot rophe-lateralsklerose-als; Stand: 16/07/2018

[2] Oliver D. Motor Neurone Disease. A family affair. 3rd ed. London: Sheldon Press; 2011.

[3] Machtoub L, Kasugai Y. Amyotrophic Lateral Sclerosis. Advances and Perspectives of Neuro-Nanomedicine. Taylor \& Boca Raton: Francis Group; 2016.

[4] Gastl R, Ludolph A. Amyotrophe Lateralsklerose. Nervenarzt 2007; 12: 1449-1459.

[5] Chio A, Gauthier A, Vignola A et al. Caregiver time use in ALS. J Neurol 2006; 67: 902-904.

[6] Grün H, Laue K, Stallbohm M. ALS: Amyotrophe Lateralsklerose. Ein Ratgeber für Betroffene, Angehörige und (Sprach-)Therapeuten. Idstein: Schulz-Kirchner Verlag; 2011.

[7] Burkhardt C, Neuwirth C, Weber M. Verhaltensänderungen bei der Amyotrophen Lateralsklerose. Hintergrund Swiss Med Forum Schweiz Medizin-Forum 2016; 10: 249-252.

[8] Merrilees J, Klapper J, Murphy J et al. Cognitive and behavioral challenges in caring for patients with frontotemporal dementia and amyotrophic lateral sclerosis. Amyotroph Lateral Scler 2010; 11: 298-302.

[9] Woolley S, Moore D, Katz J. Insight in ALS: Awareness of behavioural change in patients with and without FTD. Amyotroph Lateral Scler 2010; 11: 1-5.

[10] Pagini F, Phillips D, Bosma C et al. Mindfulness as a protective factor for the burden of caregivers of amyotrophic lateral sclerosis patients. J Clin Psychol 2016; 72: 101-111. Epub 2015 Oct 20. doi:10.1002/jclp.22235. 
[11] Andrews S, Pavlis A, Staios M et al. Which behaviours? Identifying the most common and burdensome behaviour changes in amyotrophic lateral sclerosis. Psychol Health Med 2016; 22: 483-492.

[12] Bock M, Duong Y, Kim A et al. Cognitive-behavioral changes in amyotrophic lateral sclerosis: Screening prevalence and impact on patients and caregivers. Amyotroph Lateral Scler Frontotemporal Degener 2016; 17: 366-373.

[13] Burke T, Elamin M, Galvin M et al. Caregiver burden in amyotrophic lateral sclerosis: A cross-sectional investigation of predictors. J Neurol 2015; 262: 1526-1532.

[14] Caga J, Hsieh S, Highton-Williamson E et al. The burden of apathy for caregivers of patients with amyotrophic lateral sclerosis. Amyotroph Lateral Scler Frontotemporal Degener 2018; 19: 599-605.

[15] Chio A, Vignola A, Mastro E et al. Neurobehavioral symptoms in ALS are negatively related to caregivers' burden and quality of life. Eur J Neurol 2010; 17: 1298-1303.

[16] Cui B, Cui LY, Liu MS et al. Behavioral symptoms in motor neurone disease and their negative impact on caregiver burden. Chin Med J 2015; 17: 2295-2300.

[17] Lillo P, Mioshi E, Hodges J. Caregiver burden in amyotrophic lateral sclerosis is more dependent on patients' behavioral changes than physical disability: A comparative study. BioMed Cent Neurol 2012; 12: 156-162.

[18] Watermeyer T, Brown R, Sidle $K$ et al. Impact of disease, cognitive and behavioural factors on caregiver outcome in amyotrophic lateral sclerosis. Amyotroph Lateral Scler Frontotemporal Degener 2015; 16: 316323.

[19] Tremolizzo L, Pellegrini A, Susani E et al. Behavioural but not cognitive impairment is a determinant of caregiver burden in amyotrophic lateral sclerosis. Eurn Neurol 2016; 75: 191-194.

[20] Goldstein L, Atkins L, Landau S et al. Predictors of psychological distress in carers of people with amyotrophic lateral sclerosis: A longitudinal study. Psychol Med 2006; 36: 1-11.
[21] Hecht M, Graesel E, Tigges S et al. Burden of care in amyotrophic lateral slerosis. Palliat Med 2003; 17: 327-333.

[22] Houseman G, Walsh S, Brothers A et al. Frontotemporal degeneration in the patient with amyotrophic lateral sclerosis: Helping the caregiver cope. J Neurosci Nurs 2013; 45: 96-100.

[23] Wicks $P$, Frost ]. ALS patients request more information about cognitive symptoms. Eur J Neurol 2008; 15: 495-500.

[24] Weisser F, Bristowe K, Jackson D. Experiences of burden, needs, rewards and resilience in family caregivers of people living with Motor Neurone Disease / Amyotrophic Lateral Sclerosis: A secondary thematic analysis of qualitative interviews. J Palliat Med 2015; 29: 737-745.

[25] Galvin M, Corr B, Madden C et al. Caregiving in ALS - a mixed methods approach to the study of burden. BioMed Cent Palliative Care 2016; 15 : $1-12$.

[26] Rabkin J, Albert S, Rowland L et al. How common is depression among ALS caregivers? A longitudinal study. Amyotroph Lateral Scler 2009; 10: 448-455.

[27] Pagini F, Rossi G, Lunetta C et al. Burden, depression, and anxiety in caregivers of people with amyotrophic lateral sclerosis. Psychol Health Med 2010; 15: 685-693.

[28] Pinho A, Goncalves E. Are amyotrophic lateral sclerosis caregivers at higher risk for health problems. Acta Med Port 2016; 29: 56-62.

[29] Ray R, Street A. Non-finite loss and emotional labour: Family caregivers' experiences of living with motor neurone disease. J Nurs Healthc Chronic Illn 2007; 16: 35-43.

[30] Gauthier A, Vignola A, Calvo A et al. A longitudinal study on quality of life and and depression in ALS patient-caregiver couples. J Neurol 2007; 68: 923-926. 